\title{
Detection of SARS-CoV-2 variants by Abbott molecular, antigen, and serological tests
}

3 Mary A Rodgers ${ }^{* 1}$, Rahul Batra ${ }^{2}$, Luke B Snell ${ }^{2}$, David Daghfal ${ }^{1}$, Richard Roth ${ }^{3}$, Shihai Huang ${ }^{4}$,

4 Stephen Kovacs ${ }^{5}$, Gaia Nebbia², Sam Douthwaite², Gavin A Cloherty ${ }^{1}$

$6 \quad{ }^{1}$ Abbott Diagnostics, Abbott Park, IL 60064

$7 \quad 2$ Guy's and St Thomas' NHS Foundation, London, UK

$8 \quad{ }^{3}$ Abbott Rapid Diagnostics, San Diego, CA

$9 \quad{ }^{4}$ Abbott Molecular Diagnostics, Des Plaines, IL

$10 \quad{ }^{5}$ Abbott Rapid Diagnostics, Scarborough, ME

12 *Corresponding author: Mary A Rodgers, 100 Abbott Park Rd, Abbott Park, IL 60064

\section{Highlights:}

- $\quad$ Abbott SARS-CoV-2 molecular and antigen assays detect B.1.1.7, B.1.351, and P.1 variants

- Abbott SARS-CoV-2 antibody assays detect B.1.1.7 antibodies in recovered patient sera 
medRxiv preprint doi: https://doi.org/10.1101/2021.04.24.21256045; this version posted April 26, 2021. The copyright holder for this preprint (which was not certified by peer review) is the author/funder, who has granted medRxiv a license to display the preprint in perpetuity.

It is made available under a CC-BY 4.0 International license.

\section{Abstract}

22 Background: Viral diversity presents an ongoing challenge for diagnostic tests, which need to

23 accurately detect all circulating variants. The Abbott Global Surveillance program monitors

24 severe acute respiratory syndrome coronavirus-2 (SARS-CoV-2) variants and their impact on

25 diagnostic test performance.

26 Objectives: To evaluate the capacity of Abbott molecular, antigen, and serologic assays to

27 detect the SARS-CoV-2 B.1.1.7, B.1.351 and the P.1 variants.

28 Study design: Virus variant culture stock dilutions (B.1.1.7, BEI NR-54011; B.1.351, BEI NR-

2954008 and 54009; P.1, BEI NR-54982) and clinical samples from patients with confirmed

30 B.1.1.7 variant infection were run on the Abbott ID NOW COVID-19, m2000 RealTime SARS-

31 CoV-2, Alinity m SARS-CoV-2, and Alinity m Resp-4-Plex molecular assays; the BinaxNOW

32 COVID-19 Ag Card and Panbio COVID-19 Ag Rapid Test Device; and the ARCHITECT/Alinity i

33 SARS-CoV-2 IgG and AdviseDx IgM assays, Panbio COVID-19 IgG assay, and

34 ARCHITECT/Alinity i AdviseDx SARS-CoV-2 lgG II assay.

Results: Cultured virus stocks and B.1.1.7 clinical samples were detected with molecular, antigen, and serologic assays in the expected ranges, confirming in silico predictions. The ratio between genome equivalents (GE) and calculated median tissue culture infectious dose $\left(\mathrm{TCID}_{50}\right)$ were 31 - to 83-fold higher for B.1.1.7 cultures compared to B.1.351 and P.1 cultures, demonstrating that GE are more consistent units between cultures than TCID 50 .

Conclusions: Abbott molecular and antigen assays effectively detect B.1.1.7, B.1.351, and

P.1 variant infections and Abbott serologic assays detect B.1.1.7 antibodies in patient sera. compare detection of variants.

Keywords: SARS-CoV-2, diagnostics, B.1.1.7 variant, B.1.351 variant, P.1 variant 
medRxiv preprint doi: https://doi.org/10.1101/2021.04.24.21256045; this version posted April 26, 2021. The copyright holder for this preprint (which was not certified by peer review) is the author/funder, who has granted medRxiv a license to display the preprint in perpetuity.

It is made available under a CC-BY 4.0 International license .

\section{1. Background}

47 As viruses continue to evolve, diagnostic tests must keep pace to ensure accurate detection of

48 all circulating variants. While assay design can mitigate the impact of viral diversity, assay

49 performance must be continually monitored through variant testing and molecular surveillance.

50 To meet this challenge, the Abbott Global Surveillance program has been tracking the

51 sequences of severe acute respiratory syndrome coronavirus-2 (SARS-CoV-2) variants that

52 have emerged throughout the pandemic, including three major lineages that have spread

53 globally: B.1.1.7, B.1.351, and P.1. The B.1.1.7 lineage was first identified in the United

54 Kingdom and carries spike mutations that have been linked to increased transmissibility,

55 including N501Y [1-3]. The B.1.351 lineage was first identified in South Africa and has since spread to more than a dozen countries, with initial reports indicating that this variant can escape neutralizing antibodies [4-6]. The P.1 variant was first reported in Japan as a branch of the B.1.128 lineage, but was subsequently traced back to Brazil. The P.1 variant contains similar mutations in the spike gene as the B.1.1.7 and B.1.351 variants, suggesting a convergence of SARS-CoV-2 spike mutations that may increase transmissibility and risk of reinfection [7]. While spike gene mutations primarily define all three of the major variant lineages, the presence of additional mutations throughout the genome warrant further examination for potential impact on diagnostic assay performance.

\section{Objective}

The goal of this study was to evaluate the performance of Abbott SARS-CoV-2 molecular,

67 antigen, and serological assays to detect SARS-CoV-2 B.1.17, B.1.351, and P.1 variants and B.1.1.7 antibodies. 
medRxiv preprint doi: https://doi.org/10.1101/2021.04.24.21256045; this version posted April 26, 2021. The copyright holder for this preprint (which was not certified by peer review) is the author/funder, who has granted medRxiv a license to display the preprint in perpetuity.

It is made available under a CC-BY 4.0 International license .

\section{Study design}

B.1.1.7, B.1.351, and P.1 lineage sequences were obtained from GISAID [8] for in silico comparison to assay target sequences. Virus cultures for the B.1.1.7 variant (BEI NR-54011) [9], the B.1.351 variant (BEI NR-54008, NR-54009) [10, 11], and the P.1 variant (BEI NR-54982) [12] were heat inactivated at $65^{\circ} \mathrm{C}$ for 30 minutes and diluted for testing with the Abbott ID NOW COVID-19, m2000 RealTime SARS-CoV-2, Alinity m SARS-CoV-2, and Alinity m Resp-4-Plex molecular assays; and the BinaxNOW COVID-19 Ag Card and Panbio COVID-19 Ag Rapid Test Device. Antigen testing was performed by pipetting the virus stock dilution directly onto a sterile swab and then following the manufacturer's instructions for use.

Anonymized leftover patient nasopharyngeal swab samples in viral transport media (VTM), collected at the point of discard from inpatients at Guys' and St Thomas' Hospital in London (UK Research Ethics Committee 20/SC/0310), were sequenced using the ARTIC protocol [13] on Nanopore instruments to determine infection with the B.1.1.7 variant, then run neat or diluted 562.5x before molecular testing on the Abbott ID NOW COVID-19, m2000 RealTime SARS-CoV2, Alinity m SARS-CoV-2, and Alinity m Resp-4-Plex. Matched serum samples from the same patients were used for serologic testing with the ARCHITECT/Alinity i SARS-CoV-2 IgG and AdviseDx IgM assays, Panbio COVID-19 lgG assay, and ARCHITECT/Alinity i AdviseDx SARSCoV-2 IgG II assay. All molecular and serological assays were performed per the manufacturer's instructions for use.

\section{Results}

Initial in silico examination of B.1.1.7, B.1.351, or P.1 sequences revealed no lineage-defining mutations of concern for the performance of Abbott assays targeting the RDRP region (ID NOW COVID-19, m2000 RealTime SARS-CoV-2, Alinity m SARS-CoV-2, Alinity m Resp-4-Plex), nucleocapsid region (m2000 RealTime SARS-CoV-2, Alinity m SARS-CoV-2, Alinity m Resp-4- 
medRxiv preprint doi: https://doi.org/10.1101/2021.04.24.21256045; this version posted April 26, 2021. The copyright holder for this preprint (which was not certified by peer review) is the author/funder, who has granted medRxiv a license to display the preprint in perpetuity.

It is made available under a CC-BY 4.0 International license .

98 Plex, BinaxNOW COVID-19 Ag Card, Panbio COVID-19 Ag Rapid Test Device, 99 ARCHITECT/Alinity i SARS-CoV-2 IgG, and Panbio COVID-19 lgG), and spike region 100 (ARCHITECT/Alinity i AdviseDx SARS-CoV-2 IgM, ARCHITECT/Alinity i AdviseDx SARS- CoV1012 lgG II). To evaluate these predictions for Abbott's molecular and antigen assays, dilution series 102 of heat-inactivated B.1.1.7 (BEI NR-54011) [9], B.1.351 (BEI NR-54008, NR-54009) [10, 11], and 103 P.1 (BEI NR-54982) [12] virus cultures were tested on each assay. Multiple dilutions were 104 detected with m2000, Alinity m, ID NOW, BinaxNOW, and Panbio Ag assays in the expected 105 ranges previously observed with other strains (Tables 1-3) [14, 15]. These results confirm the in silico predictions and are consistent with recent Panbio Ag evaluations of the B.1.1.7 and B.1.351 lineages $[16,17]$.

Considerable variability was observed in the ratio between genome equivalents (GE) quantitated with a standard curve and the calculated median tissue culture infectious dose $\left(T_{C} I_{50}\right)$ for each virus isolate stock (Table 4). The GE/TCID 50 ratios were 23 to 102-fold higher on average for the B.1.1.7 culture than either of the B.1.351 stocks or the P.1 stock. This difference should be taken into account when evaluating assay performance with SARS-CoV-2 virus cultures and highlights the need for standardized comparisons in units that are independent of virus culture conditions, which can vary between preparations.

117 Further evaluation was performed with leftover nasopharyngeal swab samples in VTM and 118 matched serum samples from 20 patients with sequence confirmed B.1.1.7 infections. Nineteen 119 VTM samples were run on the m2000 and both Alinity m SARS-CoV-2 assays; the sample 120 volume from patient \#19 was not sufficient for any molecular testing. The B.1.1.7 variant was 121 detected in all 19 samples by the $m 2000$ and both Alinity m assays and was detected in all 13 122 samples with sufficient volume by the Alinity Resp-4-Plex assay (Table 1). Three samples had 123 sufficient volume for testing with ID NOW; the B.1.1.7 variant was detected in all three (Table 2). 
medRxiv preprint doi: https://doi.org/10.1101/2021.04.24.21256045; this version posted April 26, 2021. The copyright holder for this preprint (which was not certified by peer review) is the author/funder, who has granted medRxiv a license to display the preprint in perpetuity.

It is made available under a CC-BY 4.0 International license .

125 Serological assays were run with serum collected from the same B.1.1.7 patients 15-26 days 126 post symptom onset (Table 4). Notably, three immune-compromised patients (\#2, \#4, and \#10)

127 were included in the panel. Antibodies for patients \#2 and \#4 were detected by four assays 128 (ARCHITECT and Alinity i IgM and IgG II), but not by ARCHITECT, Alinity i, or Panbio IgG 129 assays. Patient \#10 had cancer and did not have a detectable antibody response at 15 days post symptom onset by any assay. Excluding immune-compromised patients, antibodies were detected in 100\% (17/17) of patients by ARCHITECT/Alinity i IgM and IgG II assays and were detected in 94\% (16/17) of patients by ARCHITECT/Alinity i, and Panbio lgG assays (Table 5).

\section{Discussion}

Viral diversity will continue to challenge diagnostic tests as new SARS-CoV-2 strains arise and spread globally. Of particular interest are the B.1.1.7, B.1.351, and P.1 lineages that have been infections and B.1.1.7 antibodies.

In comparison to other RNA viruses, SARS coronaviruses maintain a higher rate of fidelity during replication due to an encoded proofreading mechanism [18]. Indeed, SARS-CoV-2 variants are typically defined by a pattern of mutations totaling $<30$ positions per genome $(0.1 \%$ divergence $)$, which is much lower than the divergence of $30 \%$ or more between HCV genotypes, for example $147[8,19,20]$. In particular, antibody assays are less prone to impact due to the redundant nature 148 of polyclonal immune responses as illustrated by the detection of spike antibodies in the sera of 149 B.1.1.7 patients (Table 4). This observation is consistent with recent studies reporting that 
medRxiv preprint doi: https://doi.org/10.1101/2021.04.24.21256045; this version posted April 26, 2021. The copyright holder for this preprint (which was not certified by peer review) is the author/funder, who has granted medRxiv a license to display the preprint in perpetuity.

It is made available under a CC-BY 4.0 International license .

150 polyclonal antibodies in convalescent plasma bind the spike protein of SARS-CoV-2 B.1.1.7 and

151 B.1.351 variants with similar binding kinetics despite variable capacity to neutralize the virus [21,

152 22]. Future studies should continue to explore the potential impact of SARS-CoV-2 sequence 153 variation on diagnostic tests.

Acknowledgements: This study was funded by Abbott Laboratories.

\section{References}

[1] Investigation of novel SARS-COV-2 variant: Variant of Concern 202012/01. [Updated March

11, 2021]. https://www.gov.uk/government/publications/investigation-of-novel-sars-cov-2-

variant-variant-of-concern-20201201 Accessed March 15, 2021.

[2] Volz E, Mishra S, Chand M, Barrett JC, Johnson R, Geidelberg L, et al. Transmission of

SARS-CoV-2 lineage B.1.1.7 in England: Insights from linking epidemiological and genetic

[3] Rambaut A, Loman N, Pybus O, Barclay W, Barrett J, Carabelli A, et al. Preliminary

genomic characterisation of an emergent SARS-CoV-2 lineage in the UK defined by a novel

set of spike mutations. [Updated Dec 2020]. https://virological.org/t/preliminary-genomic-

169 [4] Diamond M, Chen R, Xie X, Case J, Zhang X, VanBlargan L, et al. SARS-CoV-2 variants show resistance to neutralization by many monoclonal and serum-derived polyclonal antibodies. Res Sq. 2021. doi: 10.21203/rs.3.rs-228079/v1

172 [5] Tada T, Dcosta BM, Samanovic-Golden M, Herati RS, Cornelius A, Mulligan MJ, et al.

173 Neutralization of viruses with European, South African, and United States SARS-CoV-2 variant 174 spike proteins by convalescent sera and BNT162b2 mRNA vaccine-elicited antibodies. bioRxiv. 175 2021. doi: $10.1101 / 2021.02 .05 .430003$ 
medRxiv preprint doi: https://doi.org/10.1101/2021.04.24.21256045; this version posted April 26, 2021. The copyright holder for this preprint (which was not certified by peer review) is the author/funder, who has granted medRxiv a license to display the preprint in perpetuity.

It is made available under a CC-BY 4.0 International license .

176 [6] Tegally H, Wilkinson E, Giovanetti M, Iranzadeh A, Fonseca V, Giandhari J, et al.

177 Emergence and rapid spread of a new severe acute respiratory syndrome-related coronavirus

1782 (SARS-CoV-2) lineage with multiple spike mutations in South Africa. MedRxiv. 2020. doi:

$179 \quad 10.1101 / 2020.12 .21 .20248640$

180 [7] Faria N, Claro IM, Candido D, Franco LAM, Andrade PS, Coletti TM, et al. Genomic

181 characterisation of an emergent SARS-CoV-2 lineage in Manaus: preliminary findings.

182 [Updated January 12, 2021]. https://virological.org/t/genomic-characterisation-of-an-emergent-

183 sars-cov-2-lineage-in-manaus-preliminary-findings/586. Accessed April 6, 2021.

184 [8] Elbe S, Buckland-Merrett G. Data, disease and diplomacy: GISAID's innovative contribution

185 to global health. Glob Chall. 2017;1:33-46. doi: 10.1002/gch2.1018

186 [9] The following reagent was deposited by Centers for Disease Control and Prevention and

187 obtained through BEI Resources, NIAID, NIH: SARS-Related Coronavirus 2, Isolate

188 USA/CA_CDC_5574/2020,NR-54011.

189 [10] The following reagent was obtained through BEI Resources, NIAID, NIH: SARS-Related

190 Coronavirus 2, Isolate hCoV-19/South Africa/KRISP-EC-K005321/2020, NR-54008, contributed

191 by Alex Sigal and Tulio de Oliveira.

192 [11] The following reagent was obtained through BEI Resources, NIAID, NIH: SARS-Related

193 Coronavirus 2, Isolate hCoV-19/South Africa/KRISP-K005325/2020, NR-54009, contributed by

194 Alex Sigal and Tulio de Oliveira.

195 [12] The following reagent was obtained through BEI Resources, NIAID, NIH: SARS-Related

196 Coronavirus 2, Isolate hCoV-19/Japan/TY7-503/2021, NR-54982, contributed by M Takayama-

197 Ito.

198 [13] Artic Network. SARS-CoV-2. [Updated April 14, 2021]. https://artic.network/ncov-2019.

199 Accessed April 14, 2021. 
medRxiv preprint doi: https://doi.org/10.1101/2021.04.24.21256045; this version posted April 26, 2021. The copyright holder for this preprint (which was not certified by peer review) is the author/funder, who has granted medRxiv a license to display the preprint in perpetuity.

It is made available under a CC-BY 4.0 International license .

200 [14] Fung B, Gopez A, Servellita V, Arevalo S, Ho C, Deucher A, et al. Direct comparison of

201 SARS-CoV-2 analytical limits of detection across seven molecular assays. J Clin Microbiol.

202 2020;58. doi: 10.1128/JCM.01535-20

203 [15] Pilarowski G, Lebel P, Sunshine S, Liu J, Crawford E, Marquez C, et al. Performance

204 characteristics of a rapid SARS-CoV-2 antigen detection assay at a public plaza testing site in

205 San Francisco. J Infect Dis. 2021. doi: 10.1093/infdis/jiaa802

206 [16] SARS-CoV-2 Lateral Flow Antigen Tests Evaluation of VUI-20201201. [Updated

207 December 23, 2020]. https://www.gov.uk/government/publications/sars-cov-2-lateral-flow-

208 antigen-tests-evaluation-of-vui-20201201/sars-cov-2-lateral-flow-antigen-tests-evaluation-of-

209 vui-20201201. Accessed March 15, 2021.

210 [17] Akingba OL, Sprong K, Hardie DR. Field performance evaluation of the PanBio rapid

211 SARS-CoV-2 antigen assay in an epidemic driven by 501Y.v2 (lineage B.1.351) in the Eastern

212 Cape, South Africa. MedRxiv. 2021. doi: 10.1101/2021.02.03.21251057

213 [18] Sevajol M, Subissi L, Decroly E, Canard B, Imbert I. Insights into RNA synthesis, capping,

214 and proofreading mechanisms of SARS-coronavirus. Virus Res. 2014;194:90-9. doi:

$215 \quad 10.1016 / j . v i r u s r e s .2014 .10 .008$

216 [19] Hodcroft E. [Updated April 12, 2021]. https://covariants.org. Accessed April 14, 2021.

217 [20] Petruzziello A, Marigliano S, Loquercio G, Cozzolino A, Cacciapuoti C. Global

218 epidemiology of hepatitis C virus infection: An up-date of the distribution and circulation of

219 hepatitis C virus genotypes. World J Gastroenterol. 2016;22:7824-40. doi:

$220 \quad 10.3748 /$ wjg.v22.i34.7824

221 [21] Piccoli L, Park YJ, Tortorici MA, Czudnochowski N, Walls AC, Beltramello M, et al.

222 Mapping Neutralizing and Immunodominant Sites on the SARS-CoV-2 Spike Receptor-Binding

223 Domain by Structure-Guided High-Resolution Serology. Cell. 2020;183:1024-42 e21. doi:

$224 \quad 10.1016 /$ j.cell.2020.09.037 
medRxiv preprint doi: https://doi.org/10.1101/2021.04.24.21256045; this version posted April 26, 2021. The copyright holder for this preprint (which was not certified by peer review) is the author/funder, who has granted medRxiv a license to display the preprint in perpetuity.

It is made available under a CC-BY 4.0 International license .

225 [22] Planas D, Bruel T, Grzelak L, Guivel-Benhassine F, Staropoli I, Porrot F, et al. Sensitivity

226 of infectious SARS-CoV-2 B.1.1.7 and B.1.351 variants to neutralizing antibodies. Nat Med.

227 2021. doi: 10.1038/s41591-021-01318-5

228 
medRxiv preprint doi: https://doi.org/10.1101/2021.04.24.21256045; this version posted April 26, 2021. The copyright holder for this preprint (which was not certified by peer review) is the author/funder, who has granted medRxiv a license to display the preprint in perpetuity.

Tables

230

231 Table 1. m2000 and Alinity m SARS-CoV-2 Assay Results With B.1.1.7, B.1.351, and P.1 Virus

232 Culture Dilutions and B.1.1.7 Clinical Samples

\begin{tabular}{|c|c|c|c|c|c|c|}
\hline & Sample ID+ & $\mathrm{TCID}_{50} / \mathrm{mL}$ & $\begin{array}{l}\log \\
\mathrm{GE} / \mathrm{mL}^{*}\end{array}$ & $\begin{array}{c}\text { RealTime } \\
\text { SARS-CoV-2 }\end{array}$ & $\begin{array}{c}\text { Alinity m } \\
\text { SARS-CoV-2 }\end{array}$ & $\begin{array}{c}\text { Alinity m } \\
\text { Resp-4-Plex }\end{array}$ \\
\hline \multirow{23}{*}{ 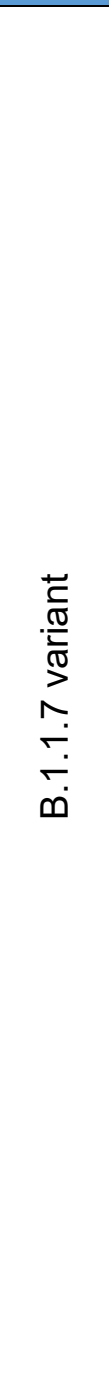 } & $\begin{array}{c}\text { BEI NR-54011 } \\
\text { Dilution } 1\end{array}$ & 28 & 6.25 & 4/4 Detected & 4/4 Detected & 4/4 Detected \\
\hline & $\begin{array}{c}\text { BEI NR-54011 } \\
\text { Dilution } 2\end{array}$ & 2.8 & 5.19 & 4/4 Detected & 4/4 Detected & 4/4 Detected \\
\hline & $\begin{array}{c}\text { BEI NR-54011 } \\
\text { Dilution } 3\end{array}$ & 0.28 & 4.00 & 4/4 Detected & 4/4 Detected & 4/4 Detected \\
\hline & $\begin{array}{c}\text { BEI NR-54011 } \\
\text { Dilution } 4\end{array}$ & 0.028 & 2.85 & 4/4 Detected & 4/4 Detected & 4/4 Detected \\
\hline & Patient 1 & $\mathrm{NA}^{\#}$ & 6.19 & 1/1 Detected & 1/1 Detected & QNS $^{+}$ \\
\hline & Patient 2 & $\mathrm{NA}^{\#}$ & 6.26 & 1/1 Detected & 1/1 Detected & QNS $^{+}$ \\
\hline & Patient 3 & $\mathrm{NA}^{\#}$ & 5.32 & 1/1 Detected & 1/1 Detected & QNS $^{+}$ \\
\hline & Patient 4 & $\mathrm{NA}^{\#}$ & 4.98 & 1/1 Detected & 1/1 Detected & 1/1 Detected \\
\hline & Patient 5 & $\mathrm{NA}^{\#}$ & 4.80 & 1/1 Detected & 1/1 Detected & 1/1 Detected \\
\hline & Patient 6 & $\mathrm{NA}^{\#}$ & 4.51 & 1/1 Detected & 1/1 Detected & 1/1 Detected \\
\hline & Patient 7 & $\mathrm{NA}^{\#}$ & 3.94 & 1/1 Detected & 1/1 Detected & 1/1 Detected \\
\hline & Patient 8 & $\mathrm{NA}^{\#}$ & 3.72 & 1/1 Detected & 1/1 Detected & 1/1 Detected \\
\hline & Patient 9 & $\mathrm{NA}^{\#}$ & 5.50 & 1/1 Detected & 1/1 Detected & 1/1 Detected \\
\hline & Patient 10 & $\mathrm{NA}^{\#}$ & 2.22 & 1/1 Detected & 1/1 Detected & 1/1 Detected \\
\hline & Patient 11 & $\mathrm{NA}^{\#}$ & 2.38 & 1/1 Detected & 1/1 Detected & QNS $^{+}$ \\
\hline & Patient 12 & $\mathrm{NA}^{\#}$ & 3.35 & 1/1 Detected & 1/1 Detected & 1/1 Detected \\
\hline & Patient 13 & $\mathrm{NA}^{\#}$ & 4.11 & 1/1 Detected & 1/1 Detected & 1/1 Detected \\
\hline & Patient 14 & $\mathrm{NA}^{\#}$ & 3.91 & 1/1 Detected & 1/1 Detected & QNS $^{+}$ \\
\hline & Patient 15 & $\mathrm{NA}^{\#}$ & 3.04 & 1/1 Detected & 1/1 Detected & 1/1 Detected \\
\hline & Patient 16 & $\mathrm{NA}^{\#}$ & 3.99 & 1/1 Detected & 1/1 Detected & 1/1 Detected \\
\hline & Patient 17 & $N A^{\#}$ & 5.49 & 1/1 Detected & 1/1 Detected & QNS $^{+}$ \\
\hline & Patient 18 & $N A^{\#}$ & 4.96 & 1/1 Detected & 1/1 Detected & 1/1 Detected \\
\hline & Patient 20 & $N^{\#}$ & 4.31 & 1/1 Detected & 1/1 Detected & 1/1 Detected \\
\hline \multirow{3}{*}{ 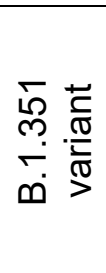 } & $\begin{array}{c}\text { BEI NR-54008 } \\
\text { Dilution } 1\end{array}$ & 28 & 4.63 & 4/4 Detected & 4/4 Detected & 4/4 Detected \\
\hline & $\begin{array}{c}\text { BEI NR-54008 } \\
\text { Dilution } 2\end{array}$ & 2.8 & 3.83 & 4/4 Detected & 4/4 Detected & 4/4 Detected \\
\hline & $\begin{array}{c}\text { BEI NR-54008 } \\
\text { Dilution } 3\end{array}$ & 0.28 & 2.51 & 4/4 Detected & 4/4 Detected & 4/4 Detected \\
\hline
\end{tabular}


medRxiv preprint doi: https://doi.org/10.1101/2021.04.24.21256045; this version posted April 26, 2021. The copyright holder for this preprint (which was not certified by peer review) is the author/funder, who has granted medRxiv a license to display the preprint in perpetuity.

It is made available under a CC-BY 4.0 International license .

\begin{tabular}{|c|c|c|c|c|c|c|}
\hline & $\begin{array}{c}\text { BEI NR-54008 } \\
\text { Dilution } 4\end{array}$ & 0.028 & 1.27 & 4/4 Detected & 4/4 Detected & 4/4 Detected \\
\hline & $\begin{array}{c}\text { BEI NR-54009 } \\
\text { Dilution } 1\end{array}$ & 28 & 4.35 & 4/4 Detected & 4/4 Detected & 4/4 Detected \\
\hline & $\begin{array}{c}\text { BEI NR-54009 } \\
\text { Dilution } 2\end{array}$ & 2.8 & 3.27 & 4/4 Detected & 4/4 Detected & 4/4 Detected \\
\hline & $\begin{array}{c}\text { BEI NR-54009 } \\
\text { Dilution } 3\end{array}$ & 0.28 & 2.11 & 4/4 Detected & 4/4 Detected & 4/4 Detected \\
\hline & $\begin{array}{c}\text { BEI NR-54009 } \\
\text { Dilution } 4\end{array}$ & 0.028 & 0.73 & 4/4 Detected & 4/4 Detected & 4/4 Detected \\
\hline \multirow{4}{*}{ 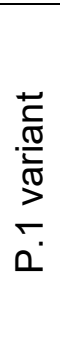 } & $\begin{array}{c}\text { BEI NR-54982 } \\
\text { Dilution } 1\end{array}$ & 28 & 4.56 & 4/4 Detected & 4/4 Detected & 4/4 Detected \\
\hline & $\begin{array}{c}\text { BEI NR-54982 } \\
\text { Dilution } 2\end{array}$ & 2.8 & 3.45 & 4/4 Detected & 4/4 Detected & 4/4 Detected \\
\hline & $\begin{array}{c}\text { BEI NR-54982 } \\
\text { Dilution } 3\end{array}$ & 0.28 & 2.39 & 4/4 Detected & 4/4 Detected & 4/4 Detected \\
\hline & $\begin{array}{c}\text { BEI NR-54982 } \\
\text { Dilution } 4\end{array}$ & 0.028 & 1.32 & 4/4 Detected & 4/4 Detected & 4/4 Detected \\
\hline
\end{tabular}

+ VTM sample quantity not sufficient (QNS) for any molecular testing for patient \#19. Several

234 indicated samples did not have sufficient quantity for testing with the Alinity m Resp-4-Plex

235 assay.

$236{ }^{*}$ Log genome equivalents $(\mathrm{GE}) / \mathrm{mL}$ were calculated from a standard curve plot of $\mathrm{TCID}_{50} \mathrm{Vs}$

237 GE with an $\mathrm{R}^{2}$ value of 0.99

238 \#NA, not applicable, TCID $_{50}$ not determined for clinical samples. 
medRxiv preprint doi: https://doi.org/10.1101/2021.04.24.21256045; this version posted April 26, 2021. The copyright holder for this preprint (which was not certified by peer review) is the author/funder, who has granted medRxiv a license to display the preprint in perpetuity.

It is made available under a CC-BY 4.0 International license .

Table 2. Rapid Molecular Assay Results With ID NOW COVID-19 with B.1.1.7, B.1.351, and

P.1 Virus Culture Dilutions and B.1.1.7 Clinical Samples

\begin{tabular}{|c|c|c|c|c|}
\hline & Sample ID & TCID $50 /$ test & Log GE/test* & IDNOW Result \\
\hline \multirow{7}{*}{ 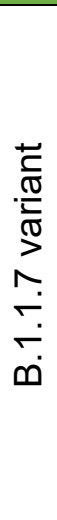 } & $\begin{array}{c}\text { BEI NR-54011 } \\
\text { Dilution } 1\end{array}$ & 5.6 & 5.55 & 3/3 Detected \\
\hline & $\begin{array}{l}\text { BEI NR-54011 } \\
\text { Dilution } 2\end{array}$ & 0.56 & 4.49 & 3/3 Detected \\
\hline & $\begin{array}{c}\text { BEI NR-54011 } \\
\text { Dilution } 3\end{array}$ & 0.056 & 3.30 & 3/4 Detected \\
\hline & $\begin{array}{c}\text { BEI NR-54011 } \\
\text { Dilution } 4\end{array}$ & 0.0112 & 2.45 & 0/3 Detected \\
\hline & Patient 4 & $N A^{\#}$ & 4.28 & 1/1 Detected \\
\hline & Patient 6 & $\mathrm{NA}^{\#}$ & 3.82 & 1/1 Detected \\
\hline & Patient 13 & $\mathrm{NA}^{\#}$ & 3.41 & 1/1 Detected \\
\hline \multirow{10}{*}{ 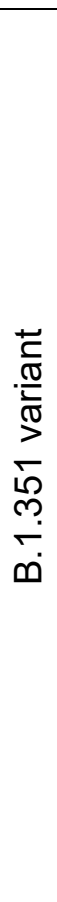 } & $\begin{array}{c}\text { BEI NR-54008 } \\
\text { Dilution } 1\end{array}$ & 5.6 & 3.94 & 3/3 Detected \\
\hline & $\begin{array}{c}\text { BEI NR-54008 } \\
\text { Dilution } 2\end{array}$ & 2.8 & 3.64 & 3/3 Detected \\
\hline & $\begin{array}{c}\text { BEI NR-54008 } \\
\text { Dilution } 3\end{array}$ & 1.4 & 3.36 & 3/3 Detected \\
\hline & $\begin{array}{c}\text { BEI NR-54008 } \\
\text { Dilution } 4\end{array}$ & 0.56 & 3.00 & 3/3 Detected \\
\hline & $\begin{array}{c}\text { BEI NR-54008 } \\
\text { Dilution } 5\end{array}$ & 0.28 & 2.76 & 1/3 Detected \\
\hline & $\begin{array}{c}\text { BEI NR-54009 } \\
\text { Dilution } 1\end{array}$ & 5.6 & 3.66 & 3/3 Detected \\
\hline & $\begin{array}{c}\text { BEI NR-54009 } \\
\text { Dilution } 2\end{array}$ & 2.8 & 3.35 & 3/3 Detected \\
\hline & $\begin{array}{c}\text { BEI NR-54009 } \\
\text { Dilution } 3\end{array}$ & 1.4 & 3.04 & 3/3 Detected \\
\hline & $\begin{array}{c}\text { BEI NR-54009 } \\
\text { Dilution } 4 \\
\end{array}$ & 0.56 & 2.63 & 2/3 Detected \\
\hline & $\begin{array}{c}\text { BEI NR-54009 } \\
\text { Dilution } 5\end{array}$ & 0.28 & 2.29 & 0/3 Detected \\
\hline \multirow{6}{*}{ 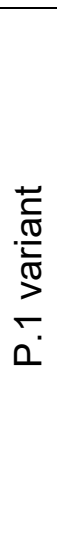 } & $\begin{array}{c}\text { BEI NR-54982 } \\
\text { Dilution } 1\end{array}$ & 5.6 & 3.86 & 3/3 Detected \\
\hline & $\begin{array}{c}\text { BEI NR-54982 } \\
\text { Dilution } 2 \\
\end{array}$ & 2.8 & 3.56 & 3/3 Detected \\
\hline & $\begin{array}{c}\text { BEI NR-54982 } \\
\text { Dilution } 3\end{array}$ & 1.4 & 3.25 & 3/3 Detected \\
\hline & $\begin{array}{c}\text { BEI NR-54982 } \\
\text { Dilution } 4\end{array}$ & 0.56 & 2.83 & 3/3 Detected \\
\hline & $\begin{array}{c}\text { BEI NR-54982 } \\
\text { Dilution } 5\end{array}$ & 0.28 & 2.49 & 3/3 Detected \\
\hline & $\begin{array}{c}\text { BEI NR-54982 } \\
\text { Dilution } 6\end{array}$ & 0.14 & 2.19 & 1/3 Detected \\
\hline
\end{tabular}

\#NA, not applicable, $\mathrm{TCID}_{50}$ not determined for clinical samples. 
medRxiv preprint doi: https://doi.org/10.1101/2021.04.24.21256045; this version posted April 26, 2021. The copyright holder for this preprint (which was not certified by peer review) is the author/funder, who has granted medRxiv a license to display the preprint in perpetuity.

It is made available under a CC-BY 4.0 International license.

242 * Log genome equivalents (GE)/test were calculated from a standard curve plot of TCID $_{50}$ Vs

243 GE with an $R^{2}$ value of 0.99 . 
medRxiv preprint doi: https://doi.org/10.1101/2021.04.24.21256045; this version posted April 26, 2021. The copyright holder for this preprint (which was not certified by peer review) is the author/funder, who has granted medRxiv a license to display the preprint in perpetuity.

It is made available under a CC-BY 4.0 International license .

Table 3. Rapid Antigen Assay Results with Virus Culture Dilutions for B.1.1.7 (BEI NR-54011),

B.1.351 (BEI NR-54008 and 54009), and P.1 (NR-54982)

\begin{tabular}{|c|c|c|c|c|c|}
\hline & Culture & TCID $50 /$ test & $\begin{array}{c}\text { Log } \\
\text { GEE/test* }\end{array}$ & $\begin{array}{c}\text { BinaxNOW } \\
\text { Result }\end{array}$ & $\begin{array}{c}\text { Panbio Ag } \\
\text { Result }\end{array}$ \\
\hline \multirow{4}{*}{ 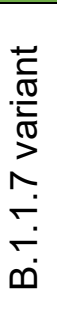 } & Neat & 560 & 7.56 & 1/1 Positive & 1/1 Positive \\
\hline & $\begin{array}{c}\text { BEI NR-54011 } \\
\text { Dilution } 1\end{array}$ & 56 & 6.56 & 4/4 Positive & 3/3 Positive \\
\hline & $\begin{array}{l}\text { BEI NR-54011 } \\
\text { Dilution } 2\end{array}$ & 28 & 6.26 & 4/4 Positive & 3/3 Positive \\
\hline & $\begin{array}{c}\text { BEI NR-54011 } \\
\text { Dilution } 3\end{array}$ & 14 & 5.96 & 3/4 Positive & 3/4 Positive \\
\hline \multirow{8}{*}{ 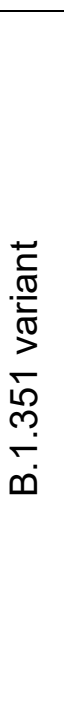 } & $\begin{array}{c}\text { BEI NR-54008 } \\
\text { Dilution } 1\end{array}$ & 1160 & 6.25 & 3/3 Detected & 3/3 Detected \\
\hline & $\begin{array}{c}\text { BEI NR-54008 } \\
\text { Dilution } 2\end{array}$ & 928 & 6.15 & 3/3 Detected & 3/3 Detected \\
\hline & $\begin{array}{c}\text { BEI NR-54008 } \\
\text { Dilution } 3\end{array}$ & 560 & 5.93 & 6/6 Detected & 3/3 Detected \\
\hline & $\begin{array}{l}\text { BEI NR-54008 } \\
\text { Dilution } 4\end{array}$ & 56 & 4.93 & 0/3 Detected & 0/3 Detected \\
\hline & $\begin{array}{l}\text { BEI NR-54009 } \\
\text { Dilution } 1\end{array}$ & 1160 & 5.98 & 3/3 Detected & 3/3 Detected \\
\hline & $\begin{array}{l}\text { BEI NR-54009 } \\
\text { Dilution } 2\end{array}$ & 928 & 5.88 & 3/3 Detected & 3/3 Detected \\
\hline & $\begin{array}{c}\text { BEI NR-54009 } \\
\text { Dilution } 3\end{array}$ & 560 & 5.66 & 6/6 Detected & 3/3 Detected \\
\hline & $\begin{array}{c}\text { BEI NR-54009 } \\
\text { Dilution } 4\end{array}$ & 56 & 4.66 & 3/3 Detected & 0/3 Detected \\
\hline \multirow{5}{*}{ 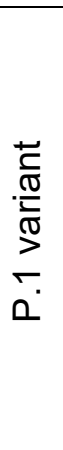 } & $\begin{array}{c}\text { BEI NR-54982 } \\
\text { Dilution } 1\end{array}$ & 1160 & 6.18 & 3/3 Detected & 3/3 Detected \\
\hline & $\begin{array}{c}\text { BEI NR-54982 } \\
\text { Dilution } 2\end{array}$ & 928 & 6.08 & 3/3 Detected & 3/3 Detected \\
\hline & $\begin{array}{c}\text { BEI NR-54982 } \\
\text { Dilution } 3\end{array}$ & 560 & 5.86 & 3/3 Detected & 3/3 Detected \\
\hline & $\begin{array}{c}\text { BEI NR-54982 } \\
\text { Dilution } 4\end{array}$ & 280 & 5.56 & 3/3 Detected & 3/3 Detected \\
\hline & $\begin{array}{l}\text { BEI NR-54982 } \\
\text { Dilution } 5\end{array}$ & 56 & 4.86 & 0/3 Detected & 0/3 Detected \\
\hline
\end{tabular}

247 GE with an $R^{2}$ value of 0.99 . 
252 Table 4. Comparison of GE*/TCID ${ }_{50}$ Ratios

\begin{tabular}{|c|c|c|c|c|c|c|c|c|c|c|c|c|}
\hline \multirow[b]{2}{*}{ Dilution } & \multirow[b]{2}{*}{ TCID50/mL } & \multicolumn{2}{|c|}{$\begin{array}{l}\text { B.1.1.7 variant } \\
\text { (NR-54011) }\end{array}$} & \multicolumn{2}{|c|}{$\begin{array}{l}\text { B.1.351 variant } \\
\text { (NR-54008) }\end{array}$} & \multicolumn{2}{|c|}{$\begin{array}{l}\text { B.1.351 variant } \\
\text { (NR-54009) }\end{array}$} & \multicolumn{2}{|c|}{$\begin{array}{l}\text { P.1 variant } \\
\text { (NR-54982) }\end{array}$} & \multicolumn{3}{|c|}{ Fold difference in GE/TCID } \\
\hline & & $\begin{array}{l}\text { Mean } \\
\text { log } \\
\text { GE } / \mathrm{mL} \\
\end{array}$ & $\begin{array}{l}\text { GE/TCID50 } \\
\text { ratio }\end{array}$ & $\begin{array}{c}\text { Mean } \\
\text { log } \\
\text { GE } / \mathrm{mL}\end{array}$ & $\begin{array}{l}\text { GE/TCID } 50 \\
\text { ratio }\end{array}$ & $\begin{array}{c}\text { Mean } \\
\text { log } \\
\text { CEE/mL }\end{array}$ & $\begin{array}{l}\text { GE/TCID50 } \\
\text { ratio }\end{array}$ & $\begin{array}{c}\text { Mean } \\
\text { log } \\
\text { GE } / m \text { L }\end{array}$ & $\begin{array}{l}\text { GE/TCID50 } \\
\text { ratio }\end{array}$ & $\begin{array}{c}\text { Ratio } \\
\text { NR-54011/ } \\
\text { NR-54008 } \\
\end{array}$ & $\begin{array}{c}\text { Ratio } \\
\text { NR-54011/ } \\
\text { NR-54009 } \\
\end{array}$ & $\begin{array}{c}\text { Ratio } \\
\text { NR-54011/ } \\
\text { NR-54982 }\end{array}$ \\
\hline 1 & 28 & 6.25 & 65189 & 4.63 & 1540 & 4.35 & 809 & 4.56 & 1296 & 42 & 81 & 50 \\
\hline 2 & 2.8 & 5.19 & 55963 & 3.83 & 2454 & 3.27 & 672 & 3.45 & 1020 & 23 & 83 & 55 \\
\hline 3 & 0.28 & 4.00 & 36169 & 2.51 & 1193 & 2.11 & 471 & 2.39 & 913 & 30 & 77 & 40 \\
\hline 4 & 0.028 & 2.85 & 25294 & 1.27 & 674 & 0.73 & 248 & 1.32 & 755 & 38 & 102 & 33 \\
\hline Average & NA & NA & 45654 & NA & 1465 & NA & 550 & NA & 996 & 31 & 83 & 46 \\
\hline
\end{tabular}


medRxiv preprint doi: https://doi.org/10.1101/2021.04.24.21256045; this version posted April 26, 2021. The copyright holder for this preprint (which was not certified by peer review) is the author/funder, who has granted medRxiv a license to display the preprint in perpetuity.

It is made available under a CC-BY 4.0 International license.

255 Table 5. SARS-CoV-2 Serology Assay Results with B.1.1.7 Clinical Samples

\begin{tabular}{|c|c|c|c|c|c|c|c|c|}
\hline $\begin{array}{c}\text { Sample } \\
\text { ID }\end{array}$ & $\begin{array}{c}\text { ARCH } \\
\operatorname{lgM} \\
(\text { Index })^{+}\end{array}$ & $\begin{array}{c}\text { Alinity } \\
\text { IgM } \\
\text { (Index) }^{+}\end{array}$ & $\begin{array}{c}\text { ARCH } \\
\text { IgG } \\
\text { (Index) }^{+}\end{array}$ & $\begin{array}{c}\text { Alinity } \\
\text { IgG } \\
\text { (Index) }^{+}\end{array}$ & $\begin{array}{c}\text { ARCH lgG } \\
\text { II } \\
\left(_{(\mathrm{AU} / \mathrm{mL})^{+}}\right.\end{array}$ & $\begin{array}{c}\text { Alinity } \\
\text { Ig G II } \\
\text { (AU/mL)+ }\end{array}$ & $\begin{array}{l}\text { Panbio } \\
\text { IgG } \\
\text { Result }\end{array}$ & $\begin{array}{c}\text { Days } \\
\text { post } \\
\text { symptom } \\
\text { onset }\end{array}$ \\
\hline Patient 1 & 18.36 & 18.68 & 6.76 & 6.97 & 13359.5 & 11595.4 & Positive & 26 \\
\hline Patient 2 & 1.22 & 1.26 & 0.22 & 0.22 & 178.5 & 189.5 & Negative & 20 \\
\hline Patient 3 & 11.68 & 12.05 & 5.62 & 6.01 & 5288.1 & 6570.1 & Positive & 19 \\
\hline Patient 4 & 11.71 & 11.04 & 0.8 & 0.87 & 149.4 & 153.7 & Negative & 25 \\
\hline Patient 5 & 6.47 & 6.58 & 5.29 & 5.44 & 5472.9 & 5468.1 & Positive & 20 \\
\hline Patient 6 & 1.31 & 1.32 & 6.6 & 6.58 & $>40000.0$ & $>40000.0$ & Positive & 16 \\
\hline Patient 7 & 14.16 & 13.51 & 6.8 & 6.98 & 12034.7 & 11191.5 & Positive & 22 \\
\hline Patient 8 & 7.41 & 7.2 & 6.39 & 6.36 & 14489.9 & 12718.3 & Positive & 21 \\
\hline Patient 9 & 15.15 & 14.86 & 4.73 & 4.82 & 2745.6 & 2603.7 & Positive & 20 \\
\hline Patient 10 & 0.03 & 0.04 & 0.02 & 0.02 & 0.9 & 0.2 & Negative & 15 \\
\hline Patient 11 & 1.27 & 1.22 & 0.22 & 0.23 & 186.4 & 186.8 & Negative & 20 \\
\hline Patient 12 & 26.79 & 26.67 & 6.62 & 6.6 & 22392.8 & 19367.7 & Positive & 23 \\
\hline Patient 13 & 5.81 & 6.06 & 4.31 & 4.33 & 1255.4 & 1183.3 & Positive & 19 \\
\hline Patient 14 & 88.25 & 88.59 & 6.67 & 6.42 & 6811.4 & 6250 & Positive & 16 \\
\hline Patient 15 & 88.27 & 88.14 & 6.66 & 6.58 & 6815.2 & 6587.5 & Positive & 21 \\
\hline Patient 16 & 88.87 & 89.11 & 6.39 & 6.5 & 6924.4 & 6401.6 & Positive & 18 \\
\hline Patient 17 & 2.09 & 2.2 & 6.23 & 6.19 & 1626.4 & 1630.5 & Positive & 17 \\
\hline Patient 18 & 91.31 & 87.56 & 6.62 & 6.55 & 7459.3 & 6330 & Positive & 19 \\
\hline Patient 19 & 4.56 & 4.58 & 7.36 & 7.31 & 2345.6 & 2274.9 & Positive & 19 \\
\hline Patient 20 & 4.55 & 4.61 & 7.18 & 7.53 & 2491.1 & 2236.1 & Positive & 20 \\
\hline
\end{tabular}

${ }^{+}$An index value of $\geq 1.4$ is positive for IgG assays, $\geq 1.0$ is positive for IgM assays, a result of

$257 \geq 50 \mathrm{AU} / \mathrm{ml}$ per $\mathrm{mL}$ is positive for lgG II assays.

258

259 